CLINICAL STUDY

\title{
Glucocorticoid treatment and impaired mood, memory and metabolism in people with diabetes: the Edinburgh Type 2 Diabetes Study
}

\author{
Rebecca M Reynolds ${ }^{1}$, Javier Labad ${ }^{1,2}$, Alison V Sears ${ }^{3}$, Rachel M Williamson ${ }^{3}$, Mark W J Strachan ${ }^{3}$, Ian J Deary ${ }^{4,5}$, \\ Gordon D O Lowe ${ }^{6}$, Jackie F Price ${ }^{5,7}$ and Brian R Walker ${ }^{1}$ on behalf of the Edinburgh Type 2 Diabetes study \\ investigators \\ ${ }^{1}$ Endocrinology Unit, University/BHF Centre for Cardiovascular Science, Queen's Medical Research Institute, University of Edinburgh, 47 Little France \\ Crescent, Edinburgh EH16 4TJ, UK, ${ }^{2}$ Hospital Psiquiatric Universitari Institut Pere Mata, Area de Recerca, Ctra. de l'Institut Pere Mata s/n, 43206 Reus, \\ Spain, ${ }^{3}$ Metabolic Unit, Western General Hospital, Edinburgh, UK, ${ }^{4}$ Department of Psychology, University of Edinburgh, Edinburgh, UK, ${ }^{5}$ Centre for \\ Cognitive Ageing and Cognitive Epidemiology, University of Edinburgh, Edinburgh, UK, ${ }^{6}$ Division of Cardiovascular and Medical Sciences, University of \\ Glasgow, Scotland, UK and ${ }^{7}$ Centre for Population Health Sciences, University of Edinburgh, Edinburgh, UK
}

(Correspondence should be addressed to R M Reynolds; Email: r.reynolds@ed.ac.uk)

\begin{abstract}
Objective: Both type 2 diabetes and glucocorticoid therapy are highly prevalent. Although people with type 2 diabetes may be more susceptible to adverse effects of glucocorticoids, and it is recommended that glucocorticoid therapy is avoided for fear of worsening glycaemic control, the extent to which this advice is followed and the consequences when glucocorticoids are prescribed are poorly documented. The aim was to assess the characteristics of people with type 2 diabetes prescribed glucocorticoids in a real-world setting and to quantify resulting adverse effects.

Design: Cross-sectional cohort study.

Methods: Cardiometabolic variables, body fat distribution, cognitive function and mood were studied in the 1066 participants of the Edinburgh Type 2 Diabetes Study, of whom 162 (15\%) were taking systemic, topical or inhaled glucocorticoids.

Results: Glucocorticoid therapy was more common in women and in smokers but was not avoided in patients with diabetic complications or cardiovascular risk factors. People taking glucocorticoids were more centrally obese with slightly higher $\mathrm{HbAlc}$ and total serum cholesterol but were no more likely to have hepatic steatosis or hypertension. Glucocorticoid treatment was associated with substantially lower mood and greater anxiety. Women taking glucocorticoid therapy were twice as likely to report depressive symptoms compared with those not taking treatment. Glucocorticoid therapy was also associated with poorer cognitive function among those with subclinical atherosclerosis, as indicated by low ankle-brachial pressure index.

Conclusions: Glucocorticoids are prescribed commonly for people with type 2 diabetes despite being associated with adverse indices of glycaemic control, cardiovascular risk factors, mood and cognitive function.
\end{abstract}

European Journal of Endocrinology 166 861-868

\section{Introduction}

Data from the UK suggest that $\sim 1 \%$ of the adult population are prescribed oral glucocorticoids (1) and a larger proportion receive inhaled or topical glucocorticoids with potential for adverse systemic effects. It is well known that acute effects of glucocorticoids may cause hyperglycaemia by increasing insulin resistance (2) and inhibiting glucose-stimulated insulin secretion from pancreatic $\beta$-cells (3). In the medium term, glucocorticoid-induced obesity and liver fat accumulation may exacerbate this metabolic dysfunction. After chronic administration, the propensity of glucocorticoids to produce hyperglycaemia, hypertension and dyslipidaemia may confer an increased risk of myocardial infarction (MI), stroke and heart failure $(4,5)$. Moreover, glucocorticoid therapy has additional adverse effects, including on mood $(6,7)$ and cognitive ability $(6,8)$. As people with type 2 diabetes are at high cardiovascular risk (9), are susceptible to depression (10) and have accelerated cognitive decline (11), and preventing cardiovascular disease is the major goal of anti-diabetic therapy, it appears logical to conclude that glucocorticoid therapy should be avoided in people with type 2 diabetes.

However, in many inflammatory diseases, glucocorticoid therapy can be difficult to avoid. Although several studies have quantified an approximately twofold 
increased risk of newly incident diabetes associated with glucocorticoid therapy $(12,13,14)$, the consequences of resorting to glucocorticoid therapy in people with established type 2 diabetes are poorly documented. As many people with type 2 diabetes are prescribed therapies to reduce cardiovascular risk, including lipid- and blood pressure-lowering agents, it is uncertain whether the adverse cardiovascular risk associated with glucocorticoid therapy is manifest in people with type 2 diabetes. Against this background, we aimed to establish whether glucocorticoid therapy is avoided in people with type 2 diabetes, in particular those most at risk of cardiovascular disease, and document the consequences when it is not avoided. As it would not be ethical to conduct a randomised controlled trial to answer these questions on glucocorticoid exposure, we used an observational study to examine the adverse associations with glucocorticoid therapy in a large well-characterised cohort of people with type 2 diabetes, the Edinburgh Type 2 Diabetes Study (ET2DS).

\section{Materials and methods}

\section{Participants and procedures}

The ET2DS is a prospective study investigating mechanisms and risk factors for diabetes-related cognitive decline and for the development and progression of micro- and macro-vascular disease in diabetes (15). One thousand and sixty-six participants aged 60-75 years were recruited at random from a comprehensive database of people with type 2 diabetes living in the Lothian region of southeast Scotland during August 2006-2007. The recruitment and study protocol have been described in detail previously (15). Briefly, subjects attended a local research clinic for physical examination including anthropometry, measurement of blood pressure, ankle-brachial index (ABI) and recording of ECG. Subjects completed questionnaires containing validated questions on demographic characteristics, recall of a doctor diagnosis of MI and angina, the WHO chest pain questionnaire (16) and cardiovascular risk factors. Cognitive ability was assessed using a comprehensive battery of validated psychometric tests, and a general cognitive ability factor, $g$, was calculated as described previously (11). The Hospital Anxiety and Depression Scale (HADS) was used to evaluate current anxiety and depressive symptoms. This scale performs well in screening for the separate dimensions of anxiety and depression in older people (17) and allows identification of cases of anxiety disorders and depression in patients from nonpsychiatric hospital clinics (18). A fasting blood sample was collected for measurement of plasma glucose, $\mathrm{HbA1c}$, total and HDL-cholesterol, liver function tests and inflammatory markers including white cell count, neutrophil count, C-reactive protein (CRP), interleukin 6 (IL6) and fibrinogen levels. Data were also collected from the Information Services Division of NHS Health Services Scotland on all medical and surgical discharges from Scottish hospitals since 1981 (SMR01 scheme). Any ICD10 or ICD9 codes for cardiovascular or cerebrovascular disease were extracted. Ischaemic heart disease was defined and categorised as described previously (19).

One year after baseline examination, participants were invited to attend again for assessment of liver structure. Nine hundred and thirty-nine subjects $(88 \%$ of the original cohort) attended after a 4-h fast for an ultrasound of the liver (20). The results were graded for hepatic steatosis using standardised criteria and validated with ${ }^{1} \mathrm{H}$ magnetic resonance spectroscopy (MRS), the non-invasive gold standard for quantification of hepatic fat (21). Non-alcoholic fatty liver disease was defined as the presence of definite hepatic steatosis on ultrasound scan (i.e. Grade 3) in the absence of a secondary cause for hepatic steatosis.

The study complied with the Declaration of Helsinki and ethical approval and written informed consent were obtained.

\section{Assessment of glucocorticoid exposure}

Subjects were asked whether they had taken glucocorticoid therapy by any route (oral, inhaled, i.m. or topical) within the last 3 months. These data were verified by manually checking through primary care records to confirm that a prescription was given.

\section{Statistical analysis}

Data were analysed using SPSS 17.0(SPSS Inc., Chicago, IL, USA). The primary analyses compared all subjects exposed to glucocorticoids by any route. Only when the primary analysis showed a statistically significant difference, a secondary analysis was performed to examine dose-response relationships by comparing those receiving glucocorticoids by topical, inhaled or systemic (oral or i.m.) routes. As there were only two subjects taking i.m. glucocorticoids, we grouped oral and i.m. glucocorticoids in the same category (equivalent to high-dose, systemic therapy).

Differences in characteristics, diabetic complications, metabolic profile and performance on the cognitive tests between those taking glucocorticoid treatment or not taking glucocorticoids were tested using unpaired $t$-tests or $\chi^{2}$ tests as appropriate. Multivariate analysis with general linear modelling (analysis of covariance (ANCOVA)) was performed including potential confounding factors of duration of diabetes, other therapies (e.g. for diabetes or lipid lowering), smoking and body mass index (BMI) as covariables as indicated. A trend analysis examining the dose-response relationship among treatment groups (not taking glucocorticoids, topical, inhaled and systemic) was performed using 
linear polynomial contrasts. We also used ANCOVA to adjust cognitive measures by several covariates including Mill Hill Vocabulary Scale, which was used as an indicator of peak prior ability and thereby afforded the estimation of lifetime cognitive change. As the distribution of several variables (Trail Making Test, HADS anxiety and depression score, $\gamma$-glutamyl transferase, CRP, IL6) was skewed, we used natural log transformation to normalise these variables. To test for gender differences in depression and anxiety scores, a stratified analysis by gender was performed. The depression scores were also categorised using a score of $\geq 8$ and odds ratios (OR) for risk of depression according to glucocorticoid therapy calculated.

Based on our previous observation of a significant interaction between circulating cortisol levels and subclinical atherosclerosis (as measured by $\mathrm{ABI}$ ) in predicting cognitive dysfunction in subjects with type 2 diabetes (11), we tested the interaction between ABI and glucocorticoid treatment in all ANCOVA analyses related to mood and cognitive measures. A stratified analysis by ABI $(<0.8$ vs $\geq 0.8)$ was carried out to test whether glucocorticoid treatment was associated with poorer cognitive performance in those with lower ABIs.

Logistic regression was used to adjust dichotomous variables (e.g. hepatic steatosis) for potential confounding factors. Glucocorticoid treatment and other covariates were included in the equation as independent variables with the enter method.

\section{Results}

\section{Characteristics of participants treated with glucocorticoids}

One hundred and sixty-two (15\%) of the subjects had taken glucocorticoid treatment within the 3 months before the study. Of these, $12 \%$ were taking topical glucocorticoids, $70 \%$ inhaled glucocorticoids and $23 \%$ oral glucocorticoids. Six per cent were taking a combination of two forms of glucocorticoid therapy and two participants had received i.m. injections. Treatment was most commonly prescribed for lung conditions (77\%), skin conditions $(30 \%)$, polymyalgia rheumatica or arthritis (14\%) and gastrointestinal conditions (2\%). Table 1 shows that those taking glucocorticoids were more likely to be female and to be current smokers. Table 2 shows that subjects taking glucocorticoid therapy had a worse inflammatory profile with significantly higher white blood cell count, neutrophil count, CRP and IL6 levels. However, there were no significant differences in likelihood of prescription of glucocorticoid therapy according to current therapies for diabetes, associated cardiovascular risk factors or current diabetic complications.

Table 1 Characteristics of participants. Data are mean (S.D.) or $n(\%)$.

\begin{tabular}{|c|c|c|c|c|c|c|}
\hline & $\begin{array}{c}\text { Not taking } \\
\text { GCs }(n=904)\end{array}$ & $\begin{array}{l}\text { Taking GCs } \\
\quad(n=162)\end{array}$ & $\begin{array}{l}\text { Topical } \\
(n=15)\end{array}$ & $\begin{array}{l}\text { Inhaled } \\
(n=107)\end{array}$ & $\begin{array}{l}\text { Oral/i.m. } \\
(n=40)\end{array}$ & $\begin{array}{l}\text { GCs vs non-GCs } \\
\boldsymbol{P} \text { value }^{\mathrm{a}}(P \text { value } \\
\left.\text { for trend analysis }{ }^{\mathrm{b}}\right)\end{array}$ \\
\hline Female gender & $428(47.3)$ & $91(56.2)$ & $8(53.3)$ & $60(56.1)$ & $23(57.5)$ & $0.038(0.039)$ \\
\hline Age at assessment (years) & $67.9(4.2)$ & $68.1(4.1)$ & $66.7(3.4)$ & $67.7(4.1)$ & $69.9(4.0)$ & 0.510 \\
\hline \multicolumn{7}{|l|}{ Smoking status } \\
\hline Current smoker & $118(13.1)$ & $30(18.5)$ & $8(53.3)$ & $32(29.9)$ & 17 (42.5) & 0.155 \\
\hline Ex-smoker & $426(47.1)$ & 75 (46.3) & 5 (33.3) & 55 (51.4) & $15(37.5)$ & \\
\hline Never smoked & $360(39.8)$ & $57(35.2)$ & $2(13.3)$ & $20(18.7)$ & $8(20)$ & \\
\hline Duration of diabetes (years) & $9.1(6.5)$ & $8.7(5.9)$ & $6.6(4.2)$ & $9.3(6.6)$ & $8.0(4.3)$ & 0.449 \\
\hline \multicolumn{7}{|l|}{ Treatment of diabetes } \\
\hline Diet alone & $169(18.7)$ & $32(19.8)$ & $2(13.3)$ & $23(21.5)$ & $7(17.5)$ & 0.865 \\
\hline Oral hypoglycaemic agents & $578(63.9)$ & $100(61.7)$ & $12(80)$ & $63(58.9)$ & $25(62.5)$ & \\
\hline Insulin \pm oral hypoglycaemic agents & $157(17.4)$ & $30(18.5)$ & $1(6.7)$ & $21(19.6)$ & $8(20)$ & \\
\hline $\begin{array}{l}\text { Lifetime history of severe hypoglycaemic } \\
\text { episodes (one or more episodes) }\end{array}$ & $84(9.5)$ & $21(13.2)$ & $2(13.3)$ & $14(13.3)$ & $5(12.8)$ & 0.154 \\
\hline Treated hypertension & $712(78.8)$ & $131(81.4)$ & $13(86.7)$ & $84(79.2)$ & $34(85.0)$ & 0.453 \\
\hline Treated dyslipidaemia & $764(84.5)$ & $133(82.1)$ & $13(86.7)$ & 87 (81.3) & $33(82.5)$ & 0.438 \\
\hline \multicolumn{7}{|l|}{ Occlusive vascular disease } \\
\hline Myocardial infarction & $123(13.6)$ & $27(16.7)$ & $3(20)$ & $14(13.1)$ & $10(25)$ & 0.302 \\
\hline Angina & $245(27.1)$ & $53(32.7)$ & $7(46.7)$ & $35(32.7)$ & $11(27.5)$ & 0.143 \\
\hline Cerebrovascular disease (stroke or TIA) & $80(8.8)$ & $13(8)$ & $1(6.7)$ & $10(9.3)$ & $2(5.0)$ & 0.732 \\
\hline Ankle-brachial index & $0.98(0.21)$ & $0.97(0.19)$ & $0.99(0.11)$ & $0.95(0.20)$ & $1.01(0.19)$ & 0.443 \\
\hline \multicolumn{7}{|l|}{ Neuropathy } \\
\hline Vibration threshold right foot (V) & $14.7(8.9)$ & $14.6(9.2)$ & $13.0(9.6)$ & $14.3(8.9)$ & $16.0(9.8)$ & 0.914 \\
\hline Vibration threshold left foot (V) & $15.0(9.1)$ & $14.1(9.3)$ & $15.1(10.7)$ & $13.6(8.7)$ & $15.1(10.3)$ & 0.294 \\
\hline Albumin/creatinine ratio $(\mathrm{mg} / \mathrm{mmol})$ & $4.9(10.0)$ & $6.6(16.1)$ & $1.4(1.4)$ & $5.4(12.1)$ & $11.0(25.3)$ & 0.244 \\
\hline
\end{tabular}

GCs, glucocorticoids; TIA, transient ischaemic attack.

${ }^{\mathrm{a}} T$-test and $\chi^{2}$ test were used to compare continuous or categorical variables between treatment groups (GCs vs non-GCs).

${ }^{b}$ Trend analysis was performed when primary analyses using $T$-test or $\chi^{2}$ tests were significant. Four groups were considered for this analysis: i) not taking GCs, ii) topical, iii) inhaled and iv) oral/i.m. For continuous variables, the linear polynomial contrast of the ANOVA was used. 
Table 2 Metabolic and anthropometric variables. Data are mean (s.D.), $n$ (\%) or median (interquartile range).

\begin{tabular}{|c|c|c|c|c|c|c|c|}
\hline & \multirow[b]{2}{*}{$\begin{array}{l}\text { Not taking } \\
\text { GCs }(n=904)\end{array}$} & \multirow[b]{2}{*}{$\begin{array}{l}\text { Taking GCs } \\
\quad(n=162)\end{array}$} & \multirow[b]{2}{*}{$\begin{array}{l}\text { Topical } \\
(n=15)\end{array}$} & \multirow[b]{2}{*}{$\begin{array}{l}\text { Inhaled } \\
(n=107)\end{array}$} & \multirow[b]{2}{*}{$\begin{array}{l}\text { Oral/i.m. } \\
(n=40)\end{array}$} & \multicolumn{2}{|c|}{ GCs vs non-GCs } \\
\hline & & & & & & $\begin{array}{l}\text { Unadjusted } \\
P \text { value }^{\mathrm{a}}\end{array}$ & $\begin{array}{l}\text { Adjusted } P \text { value } \\
\text { (adjusted } P \text { value }^{\text {b }} \\
\text { for trend }{ }^{c} \text { ) }\end{array}$ \\
\hline \multicolumn{8}{|l|}{ Metabolic variables } \\
\hline $\begin{array}{l}\text { Fasting plasma glucose } \\
(\mathrm{mmol} / \mathrm{l})\end{array}$ & $7.6(2.1)$ & $7.5(2.2)$ & $7.6(2.0)$ & $7.8(2.3)$ & $6.8(1.7)$ & 0.702 & 0.842 \\
\hline $\mathrm{HbA1c}(\%)$ & $7.4(1.1)$ & $7.5(1.4)$ & $7.3(0.8)$ & $7.5(1.4)$ & $7.7(1.4)$ & 0.079 & $0.043(0.115)$ \\
\hline $\begin{array}{l}\text { Systolic blood pressure } \\
(\mathrm{mmHg})\end{array}$ & $133.2(16.3)$ & $133.3(16.8)$ & $123.5(10.5)$ & $134.8(16.5) \quad 1$ & $132.9(18.8)$ & 0.994 & 0.907 \\
\hline $\begin{array}{l}\text { Diastolic blood pressure } \\
(\mathrm{mmHg})\end{array}$ & $69.1(9.1)$ & $68.4(8.4)$ & $65.3(4.6)$ & $68.1(4.8)$ & $68.0(4.6)$ & 0.348 & 0.422 \\
\hline $\begin{array}{l}\text { Serum total cholesterol } \\
(\mathrm{mmol} / \mathrm{l})\end{array}$ & $4.3(0.9)$ & $4.5(0.9)$ & $4.3(0.8)$ & $4.5(0.9)$ & $4.4(0.9)$ & 0.020 & 0.064 \\
\hline $\begin{array}{l}\text { Serum HDL-cholesterol } \\
(\mathrm{mmol} / \mathrm{l})\end{array}$ & $1.3(0.4)$ & $1.4(0.4)$ & $1.2(0.3)$ & $1.4(0.4)$ & $1.5(0.5)$ & $<0.001$ & $0.005(0.002)$ \\
\hline \multicolumn{8}{|l|}{ Liver variables } \\
\hline $\begin{array}{l}\text { Severe hepatic steatosis } \\
\text { on ultrasound, } n(\%)\end{array}$ & $445(55.6)$ & $88(63.3)$ & $12(85.7)$ & $54(60.7)$ & $22(61.1)$ & 0.091 & $0.181^{d}$ \\
\hline Serum ALT (U/l) & $43.6(14.6)$ & $41.3(12.6)$ & $46.2(13.4)$ & $40.7(12.6)$ & $40.9(12.1)$ & 0.070 & 0.153 \\
\hline Serum AST (U/I) & $31.4(10.4)$ & $28.4(8.4)$ & $31.8(11.7)$ & $28.3(8.3)$ & $27.5(6.8)$ & 0.001 & $0.001(0.018)$ \\
\hline Serum GGT (U/I) & $2.9(2.4-3.4)$ & $2.9(2.5-3.5)$ & $2.9(2.3-3.4)$ & $2.9(2.4-3.5)$ & $3.0(2.6-3.5)$ & 0.601 & 0.658 \\
\hline Serum bilirubin $(\mu \mathrm{mol} / \mathrm{l})$ & $10.2(5.0)$ & $9.4(4.4)$ & $9.3(3.8)$ & $9.3(4.2)$ & $9.6(5.0)$ & 0.063 & 0.181 \\
\hline Serum albumin $(\mathrm{g} / \mathrm{l})$ & 44.9 (3.2) & $44.1(3.7)$ & $44.3(4.4)$ & $44.4(3.5)$ & $43.3(4.2)$ & 0.013 & $0.020(0.025)$ \\
\hline \multicolumn{8}{|c|}{ Inflammatory variables in blood } \\
\hline IL6 (pg/ml) & $2.8(1.9-4.3)$ & $3.2(2.2-5.4)$ & $2.8(1.8-4.6)$ & $3.1(2.2-5.0)$ & $3.7(2.3-9.4)$ & 0.002 & $0.006(<0.001)$ \\
\hline C-reactive protein $(\mathrm{mg} / \mathrm{l})$ & $1.8(0.8-3.9)$ & $3.0(1.3-6.6)$ & $2.2(1.3-3.1)$ & $3.2(1.2-6.7)$ & $3.3(1.4-7.7)$ & $<0.001$ & $<0.001(0.001)$ \\
\hline $\mathrm{TNF} \alpha(\mathrm{pg} / \mathrm{ml})$ & $1.1(0.7-1.6)$ & $1.2(0.7-1.7)$ & $1.2(0.6-1.6)$ & $1.1(0.7-1.6)$ & ) $1.4(1.0-1.8)$ & 0.787 & 0.500 \\
\hline Fibrinogen $(\mu \mathrm{g} / \mathrm{l})$ & $3.6(0.7)$ & $3.8(0.8)$ & $3.5(1.1)$ & $3.8(0.8)$ & $3.9(0.7)$ & 0.064 & 0.143 \\
\hline White cell count $\left(\times 10^{9} / \mathrm{l}\right)$ & $7.1(2.3)$ & $7.8(2.3)$ & $8.6(2.4)$ & $7.5(1.9)$ & $8.5(2.8)$ & $<0.001$ & $0.001(0.024)$ \\
\hline Neutrophil count $\left(\times 10^{9} / \mathrm{l}\right)$ & $4.4(1.5)$ & $5.1(1.8)$ & $5.3(1.9)$ & $4.8(1.6)$ & $5.6(2.1)$ & $<0.001$ & $<0.001(<0.001)$ \\
\hline \multicolumn{8}{|l|}{ Anthropometric variables } \\
\hline Weight (kg) & $86.2(15.9)$ & $87.6(17.3)$ & $86.3(16.0)$ & $83.3(14.2)$ & $88.8(18.3)$ & 0.327 & 0.112 \\
\hline BMI $\left(\mathrm{kg} / \mathrm{m}^{2}\right)$ & $31.3(5.6)$ & $32.3(6.0)$ & $31.1(4.1)$ & $32.7(6.6)$ & $31.5(4.9)$ & 0.045 & 0.100 \\
\hline \multicolumn{8}{|l|}{ Waist $(\mathrm{cm})$} \\
\hline Males & $107.7(11.9)$ & $111.7(12.5)$ & $112.3(8.1)$ & $112.1(13.6) \quad 1$ & $110.5(10.9)$ & 0.009 & $0.002(0.242)$ \\
\hline Females & $105.0(13.0)$ & $108.0(14.3)$ & $103.7(13.7)$ & $109.0(15.2) \quad 1$ & $107.0(12.3)$ & 0.055 & $0.070(0.128)$ \\
\hline \multicolumn{8}{|l|}{ Hip (cm) } \\
\hline Males & $107.6(9.5)$ & $110.0(9.9)$ & $109.3(11.5)$ & $109.8(10.5) \quad 1$ & $110.8(7.7)$ & 0.054 & $0.020(0.096)$ \\
\hline Females & $113.9(13.0)$ & $114.7(15.1)$ & 111.8 (13.4) & $116.3(16.8) \quad 1$ & 111.8 (10.2) & 0.577 & 0.725 \\
\hline \multicolumn{8}{|l|}{ Waist/hip } \\
\hline Males & $1.001(0.061)$ & $1.015(0.058)$ & $1.031(0.040)$ & $1.019(0.064) 0$ & $0.996(0.043)$ & 0.046 & 0.031 \\
\hline Females & $0.924(0.070)$ & $0.944(0.071)$ & $0.928(0.075)$ & $0.941(0.065) 0$ & $0.958(0.083)$ & 0.015 & $0.010(0.005)$ \\
\hline
\end{tabular}

GCs, glucocorticoids; ALT, alanine aminotransferase; AST, aspartate transaminase; GGT, $\gamma$-glutamyl transpeptidase; IL6, interleukin 6; TNF $\alpha$, tumour necrosis factor $\alpha$.

${ }^{a} T$-test and $\chi^{2}$ test were used to compare continuous or categorical variables between treatment groups (GCs vs non-GCs).

${ }^{b}$ Analysis of covariance (ANCOVA) was used to compare GCs vs non-GCs groups while adjusting for covariates. All analyses have been adjusted for gender, age and duration of diabetes. Additional adjustments have been performed: i) glucose and $\mathrm{HbA} 1 \mathrm{c} \%$ also adjusted for anti-diabetic treatment and BMI; ii) systolic and diastolic blood pressure also adjusted for anti-hypertensive treatment and BMI; iii) total cholesterol and HDL-cholesterol also adjusted for lipidlowering treatment; iv) liver variables and inflammatory variables also adjusted for BMI; and v) BMI and anthropometric variables also adjusted for smoking. ${ }^{\circ}$ When primary ANCOVA analysis was significant, a secondary trend analysis was performed using the linear polynomial contrast of ANCOVA among the four treatment groups: i) not taking GCs, ii) topical, iii) inhaled and iv) oral/i.m.

${ }^{d}$ Logistic regression was used to explore the relationship between glucocorticoid treatment and hepatic steatosis while adjusting for covariates (age, gender, duration of diabetes and $\mathrm{BMI})$.

\section{Glucocorticoid treatment and metabolic profile}

Table 2 shows that subjects taking glucocorticoids had higher BMI and waist:hip ratios than those on no treatment. Glucocorticoid treatment was also associated with poorer glycaemic control, as demonstrated by a trend for higher $\mathrm{HbAlC}$ that became significant in multivariate analysis. Total serum cholesterol and also HDL-cholesterol were higher in those taking glucocorticoid treatment; these findings remained significant after adjustment for lipid-lowering therapy. Blood pressure did not differ according to glucocorticoid therapy, even after adjustment for anti-hypertensive therapy. The prevalence of severe hepatic steatosis on ultrasound tended to be higher in participants receiving 
glucocorticoids, but this was not statistically significant and serum levels of bilirubin, alanine transferase (ALT) and aspartate transferase (AST) tended to be lower in these subjects.

\section{Glucocorticoid treatment and mood and cognitive function}

Table 3 shows that subjects treated with glucocorticoids had higher scores on both the anxiety and depression scales of the HADS. Risk of anxiety was similar in males and females, but there was a gender difference in the risk of depression, with women taking glucocorticoid therapy having higher depression scores. Using a cut-off score on the HADS-depression scale of 8 , a valid cut-off in screening for depression (18), women taking glucocorticoid therapy were twice as likely to report symptoms of depression (OR 2.35 (1.35-4.5), $P=0.005)$ compared with women not taking glucocorticoid therapy. This association was not seen in men (OR 0.83 (0.32-2.18), $P=0.699)$.

Table 3 also shows that in univariate analyses, there were no differences between subjects taking or not taking glucocorticoid therapy in overall cognitive ability $(g)$ or in the specific cognitive domains tested. However, in the multivariate analyses, we found a significant relationship between glucocorticoid treatment and a poorer performance on the TMT with a significant interaction between ABI and glucocorticoid treatment. No significant interactions were found in analyses exploring other cognitive domains or mood status. In the stratified analysis by ABI (Table 4), glucocorticoid therapy was significantly associated with poorer cognitive function on the trail-making test, with trends for poorer overall cognitive ability $(g)$ and for poorer matrix reasoning and letter number sequencing scores, in subjects with lower ABI $(<0.8)(n=147)$. Cognitive function was not related to glucocorticoid therapy in those with normal ABI.

\section{Dose-response relationships with gluco- corticoid treatment}

For variables for which there was a difference between the glucocorticoid-treated and -untreated groups, we explored dose-response relationships by comparing groups receiving topical, inhaled or systemic therapy. In general, differences in the glucocorticoid-treated group were more obvious among those receiving systemic therapy, although differences between the topical and inhaled treatment groups were less consistent, for example with regard to white blood cell and neutrophil counts and to waist:hip ratio in men.

Table 3 Mood and cognitive measures. Data are mean (s.D.) or median (interquartile range).

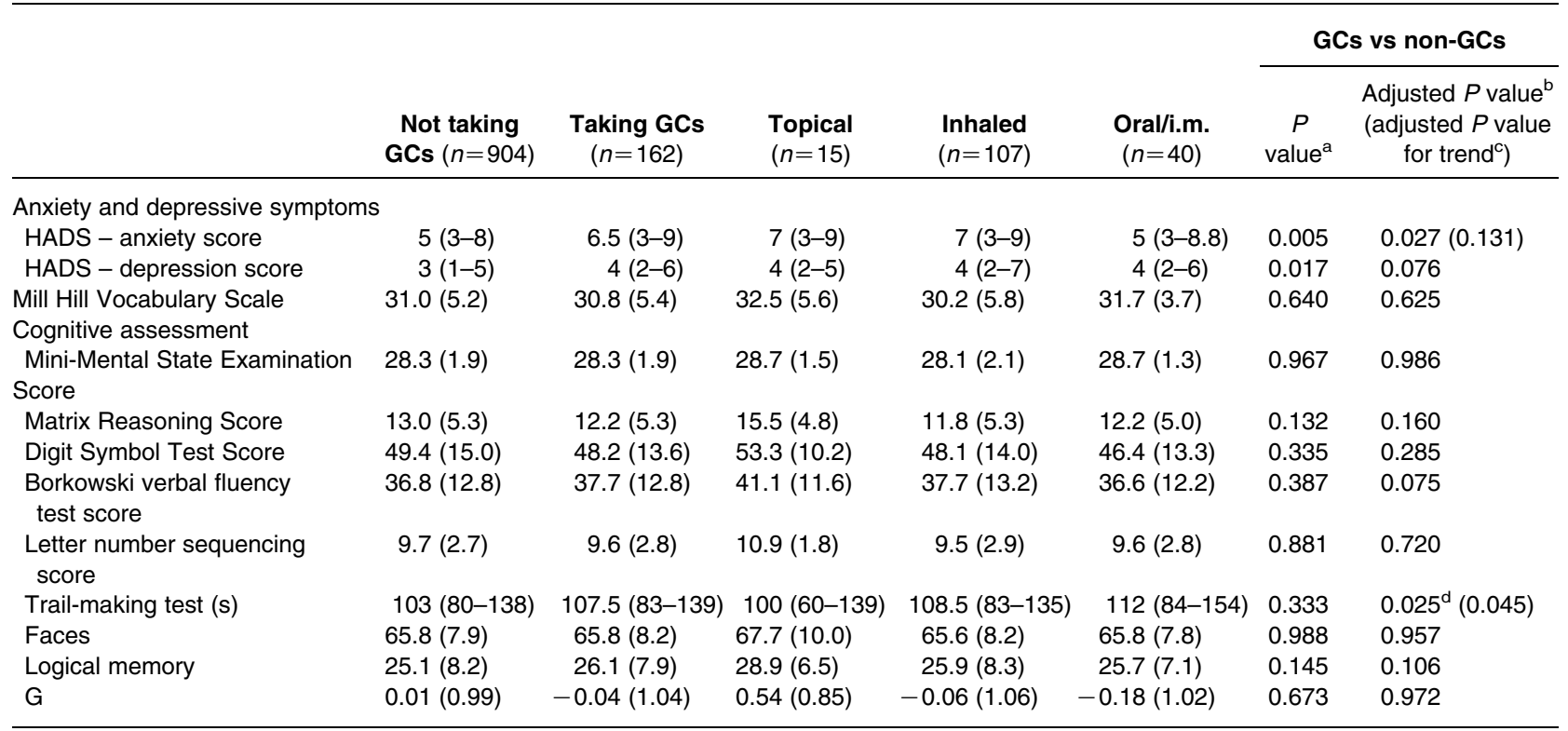

GCs, glucocorticoids; HADS, Hospital Anxiety and Depression Scale; G, general cognitive ability factor; ABI, ankle-brachial index

${ }^{a} T$-test was used to compare mood and cognition scores between treatment groups (GCs vs non-GCs).

${ }^{b}$ Analysis of covariance was used to adjust for covariates. Mood scores were adjusted for age, gender, education level, duration of diabetes, cerebrovascular disease (stroke or $\mathrm{TIA}$ ), $\mathrm{ABI}$ and antidepressant treatment. Cognitive measures were adjusted for age, gender, education level, duration of diabetes, cerebrovascular disease (stroke or TIA), ABI, Mill Hill Vocabulary Scale, HADS depression and anxiety scores.

'When primary ANCOVA analysis was significant, a secondary trend analysis was performed using the linear polynomial contrast of ANCOVA among the four treatment groups: i) not taking GCs, ii) topical, iii) inhaled and iv) oral/i.m.

${ }^{\mathrm{d}}$ A significant interaction term between glucocorticoid treatment and $\mathrm{ABI}(P=0.031)$ was included in this analysis. Other covariates significantly associated with the Trail-making test were age, duration of diabetes, cerebrovascular disease, Mill Hill Vocabulary Scale and HADS depression score. 
Table 4 Mood and cognitive measures: stratified analysis by ABI. Data are mean (S.D.) or median (interquartile range).

\begin{tabular}{|c|c|c|c|c|c|c|}
\hline & \multicolumn{3}{|c|}{ Low ABI $(<0.8)(n=147)$} & \multicolumn{3}{|c|}{ Normal ABI $(\geq 0.8)(n=912)$} \\
\hline & $\begin{array}{l}\text { Not taking GCs } \\
\quad(n=126)\end{array}$ & $\begin{array}{l}\text { Taking GCs } \\
\quad(n=21)\end{array}$ & $P$ value ${ }^{a}$ & $\begin{array}{l}\text { Not taking GCs } \\
\quad(n=772)\end{array}$ & $\begin{array}{c}\text { Taking GCs } \\
(n=140)\end{array}$ & $P$ value ${ }^{\mathrm{a}}$ \\
\hline \multicolumn{7}{|l|}{ Anxiety and depressive symptoms } \\
\hline HADS - anxiety score & $5(3-8)$ & $8(4-10)$ & 0.032 & $5(3-8)$ & $6(3-9)$ & 0.077 \\
\hline HADS - depression score & $4(2-6)$ & $6(3.5-8)$ & 0.023 & $3(1-5)$ & $4(2-6)$ & 0.030 \\
\hline Mill Hill Vocabulary Scale & $30.2(5.0)$ & $27.5(5.4)$ & 0.081 & $31.1(5.3)$ & $31.1(5.3)$ & 0.991 \\
\hline \multicolumn{7}{|l|}{ Cognitive assessment } \\
\hline Mini-Mental State Examination Score & $27.8(2.6)$ & $27.0(3.0)$ & 0.166 & $28.4(1.7)$ & $28.5(1.6)$ & 0.466 \\
\hline Matrix Reasoning Score & $12.0(5.2)$ & $10.1(5.0)$ & 0.136 & $13.1(5.3)$ & $12.5(5.2)$ & 0.923 \\
\hline Digit Symbol Test Score & $45.2(14.5)$ & $41.3(14.8)$ & 0.261 & $50.1(15.0)$ & $49.1(13.1)$ & 0.457 \\
\hline Borkowski Verbal Fluency Test Score & $36.3(13.2)$ & $34.4(14.1)$ & 0.542 & $36.7(12.7)$ & $38.0(12.4)$ & 0.260 \\
\hline Letter Number Sequencing Score & $9.5(3.0)$ & $8.2(3.0)$ & 0.092 & $9.7(2.7)$ & $9.9(2.7)$ & 0.548 \\
\hline Trail-making test $(\mathrm{s})$ & $107(84.5-135)$ & $133(114.5-133)$ & 0.002 & $103(80-139)$ & $106(81-135.5)$ & 0.870 \\
\hline Faces & $64.7(7.7)$ & $64.9(9.0)$ & 0.931 & $66.0(7.8)$ & $66.0(8.2)$ & 0.999 \\
\hline Logical memory & $24.3(8.2)$ & $26.3(9.0)$ & 0.329 & $25.2(8.2)$ & $26.0(7.7)$ & 0.265 \\
\hline $\mathrm{G}$ & $-0.19(0.93)$ & $-0.57(1.12)$ & 0.110 & $0.03(1.0)$ & $0.04(1.01)$ & 0.955 \\
\hline
\end{tabular}

$\mathrm{ABI}$, ankle-brachial pressure index; GCs, glucocorticoids; HADS, Hospital Anxiety and Depression Scale; G, general cognitive ability factor.

${ }^{\text {a }} T$-test was used to compare mood and cognition scores between treatment groups (GCs vs non-GCs).

\section{Discussion}

In this cohort study of elderly people with type 2 diabetes, glucocorticoid treatment was widely used, with nearly $14 \%$ of participants taking glucocorticoid therapy by some route. Treatment with glucocorticoids was associated with an adverse metabolic profile with increased obesity, poorer glycaemic control and higher total cholesterol (albeit also with higher atheroprotective HDL-cholesterol). Glucocorticoid therapy was also associated with lower mood, especially in women, and increased anxiety. Moreover, we found evidence that glucocorticoid therapy is independently associated with impaired cognitive function in the trail-making test among patients with subclinical atherosclerosis.

Our findings add to the literature that glucocorticoid treatment is associated with adverse cardiovascular risk among the general population $(4,5,22)$. People with type 2 diabetes are at high cardiovascular risk (9), and the majority of patients were receiving secondary preventative treatment with statins and/or blood pressure-lowering agents (15). Levels of HbAlc, cholesterol and blood pressure were within, or approaching, recommended targets (Management of Diabetes. SIGN Guideline. 2011 www.sign.ac.uk). Despite this, we still found glucocorticoid treatment was associated with poorer glycaemic control and higher total cholesterol, although the magnitude of difference between groups was small. As expected, glucocorticoid therapy was associated with increased obesity and particularly with central obesity as seen in Cushing's syndrome. However, the finding of lower levels of liver function tests in people taking glucocorticoids and relatively weak association with hepatic steatosis was surprising, as patients with spontaneous Cushing's syndrome are reported to have a high prevalence of hepatic steatosis (23). It may be relevant that the liver scans were undertaken 1 year later than the initial visit at which glucocorticoid exposure was assessed. However, when we reanalysed the data using glucocorticoid use within the 6 months before the liver scans (data not shown), the findings were no different.

Glucocorticoid excess is also a well-known cause of mood disorders, consistent with our finding that people treated with glucocorticoids had lower mood and were more anxious. The magnitude of these differences may be clinically significant; in particular, women taking glucocorticoid therapy were twice as likely to report symptoms of depression compared with those not taking glucocorticoids. Although women are at greater risk of depression than men, and this may account, in part, for a greater risk of depression with glucocorticoid treatment, there is no consistency in the literature as to whether females are more vulnerable to depressive symptoms in association with glucocorticoid therapy (6). Symptoms of depression and anxiety are common in people with diabetes (10) and are associated with poorer glycaemic and metabolic control (24). Our findings suggest that treatment with glucocorticoids is associated with symptoms of depression and anxiety in people with type 2 diabetes.

People with Cushing's syndrome have poorer memory (8), and a recent study has shown a dose-dependent inhibitory effect of glucocorticoids on emotional information processing in healthy volunteers (25). Here, we have found some evidence that glucocorticoid treatment was associated with poorer cognitive performance, mainly in processing speed. This is the first cognitive domain to show a decline with ageing, show large declines, and is an early predictor of dementia (26). We have previously shown poorer functioning on this test in association with higher endogenous cortisol levels in people with type 2 diabetes (11). In the latter study, we have also reported an interaction between circulating cortisol levels and subclinical atherosclerosis, as measured 
by $\mathrm{ABI}$, in predicting cognitive dysfunction in patients with type 2 diabetes (11). People with subclinical atherosclerosis may be more susceptible to the detrimental effects of glucocorticoids on hippocampal function (27). We have found support for this in the current study, as measurable cognitive dysfunction was restricted to patients with low ABI, particularly in the trail-making test, which is an assessment of mental flexibility. However, there was a small number of patients in this group $(n=21)$, and the lack of an association between glucocorticoid exposure and cognitive function in the whole cohort suggests that, by contrast with the effect of diabetes per se, any influence of additional glucocorticoid therapy on cognitive function is of minor clinical significance.

Given the observational study design, it is impossible to avoid potential confounding by indication. The higher levels of inflammatory indices in the blood are consistent with underlying inflammatory disease for which the patients are receiving glucocorticoid therapy, and it is possible that the underlying disease process is responsible for the association of glucocorticoid therapy with metabolic variables and mood. It was not possible to investigate the consistency of associations with glucocorticoid therapy in different disease indications because of the small number of patients available who were not being treated for obstructive pulmonary disease. However, it is unlikely that confounding by indication accounts for all the associations with glucocorticoid therapy observed here; for example, in patients with obstructive pulmonary disease, one would anticipate reduced rather than increased body fat. We also repeated all analyses to test whether inclusion of inflammatory markers (IL6, CRP) moderated the findings between glucocorticoid therapy and outcomes (data not shown) and found no change in the results. The inference that glucocorticoid therapy contributes directly to adverse metabolic outcomes is further supported by the persistence of relationships after adjustment for smoking and by the dose-response relationships with glucocorticoid exposure, although this was less apparent for symptoms of depression and anxiety.

The strengths of this study include the large sample size and the application of a battery of seven psychometric tests, which provides a relatively comprehensive and validated assessment of cognitive domains and mood states. There was also detailed phenotyping for potential confounding or mediating factors. The study population had a verified clinical diagnosis of type 2 diabetes and was representative of the wider type 2 diabetic population (elderly, community-dwelling men and women with the full spectrum of severity of type 2 diabetes, from diet-controlled to insulin-treated). Many large-scale studies on people with diabetes are restricted to those attending hospital clinics and biased towards the more severe end of the spectrum of diabetes. Although the use of multiple tests raises the possibility of multiple testing with associated false-positive results, this possibility was acknowledged by both general cognitive factors and the individual tests. The advantages of having detailed information on such a wide range of phenotypes outweigh the remaining statistical concerns. We asked all subjects about glucocorticoid treatment, including topical and inhaled glucocorticoids, as these have systemic effects. However, estimation of glucocorticoid exposure is not straightforward as glucocorticoid treatment for chronic disease is typically intermittent over many years. We verified that all subjects had received at least one prescription for glucocorticoid therapy during the 3 months before the study by manual checking of primary care prescription records, but we were unable to obtain data over a longer period to provide an estimate of cumulative glucocorticoid exposure. Unfortunately, data were not available on other important indices of the adverse effects of glucocorticoids, such as bone density.

In conclusion, a large number of our elderly subjects with type 2 diabetes were prescribed glucocorticoid therapy, and this was associated with some adverse indices of metabolic and brain function. A direct causal link cannot be proved due to the cross-sectional design of our study and prospective studies are needed. Nevertheless, clinicians should be aware that in addition to encouraging weight gain and worsening glycaemic control, glucocorticoid treatment has potential negative effects on cardiovascular risk and cognition in people with type 2 diabetes, although arguably the magnitude of these effects was surprisingly small. Perhaps more importantly, the risk of mood disturbance in patients receiving glucocorticoids deserves closer attention when deciding whether to initiate or continue glucocorticoid therapy.

\section{Declaration of interest}

The authors declare that there is no conflict of interest that could be perceived as prejudicing the impartiality of the research reported.

\section{Funding}

This study was supported by a grant from the Medical Research Council (2006-2011).

\section{Author contribution statement}

R M Reynolds designed and carried out the study, interpreted the data and wrote the manuscript. R M Reynolds is guarantor for the manuscript. J Labad carried out the statistical analysis, interpreted the data and contributed to writing the manuscript. A V Sears verified and collected the data and approved the final version of the manuscript. R M Williamson carried out the liver 'sub-study' and edited the manuscript. M W J Strachan designed and carried out the study and approved the final version of the manuscript. I J Deary designed the study, interpreted the data and contributed to the writing of the manuscript. G D O Lowe carried out the biochemical analyses and edited the manuscript. J F Price designed and carried out the study and edited the manuscript. B R Walker designed the study, interpreted the data and contributed to the writing of the manuscript. 


\section{Acknowledgements}

We acknowledge the support of the British Heart Foundation. The liver 'sub-study' was funded by Pfizer Ltd. All authors thank their co-investigators and collaborators and all the participants and staff of the Edinburgh Type 2 Diabetes Study and the staff at the Wellcome Trust Clinical Research Facility where the study was performed. J Labad received an Intensification of Research Activity grant (Programme 13SNS-INT 11/323) from the Instituto de Salud Carlos III (Spain).

\section{References}

1 van Staa TP, Leufkens HG, Abenhaim L, Begaud B, Zhang B \& Cooper C. Use of oral corticosteroids in the United Kingdom. Quarterly Journal of Medicine 200093 105-111. (doi:10.1093/ qjmed/93.2.105)

2 Andrews RC \& Walker BR. Glucocorticoids and insulin resistance: old hormones, new targets. Clinical Science 199996 513-523. (doi:10.1042/CS19980388)

3 Delaunay F, Khan A, Cintra A, Davani B, Ling Z-C, Andersson A, Ostenson CG, Gustafsson J. Efendic S \& Okret S. Pancreatic beta cells are important targets for the diabetogenic effects of glucocorticoids. Journal of Clinical Investigation $1997 \mathbf{1 0 0}$ 2094-2098. (doi:10.1172/JCI119743)

4 Souverain PC, Berard A, van Staa TP, Cooper C, Leufkens HGM \& Walker BR. Use of oral glucocorticoids and risk of cardiovascular and cerebrovascular disease in a population-based case-control study. Heart 200490 859-865. (doi:10.1136/hrt.2003.020180)

5 Wei L, MacDonald TM \& Walker BR. Taking glucocorticoids by prescription is associated with subsequent cardiovascular disease. Annals of Internal Medicine 2004141 764-770.

6 Brown ES. Effects of glucocorticoids on mood, memory, and the hippocampus. Treatment and preventive therapy. Annals of the New York Academy of Sciences 20091179 41-55. (doi:10.1111/j. 1749-6632.2009.04981.x)

7 Pariante CM. The glucocorticoid receptor: part of the solution or part of the problem? Journal of Psychopharmacology 200620 79-84. (doi:10.1177/1359786806066063)

8 Starkman MN, Giordani B, Berent S, Schork MA \& Schteingart DE. Elevated cortisol levels in Cushing's disease are associated with cognitive decrements. Psychosomatic Medicine 200163 985-993.

9 Haffner SM, Lehto S, Ronnemaa T, Pyorala K \& Laakso M Mortality from coronary heart disease in subjects with type 2 diabetes and in nondiabetic subjects with and without prior myocardial infarction. New England Journal of Medicine 1998339 229-234. (doi:10.1056/NEJM199807233390404)

10 Labad J, Price JF, Strachan MW, Fowkes FG, Ding J, Deary IJ, Lee AJ, Frier BM, Seckl JR, Walker BR \& Reynolds RM \& Edinburgh Type 2 Diabetes study investigators. Symptoms of depression but not anxiety are associated with central obesity and cardiovascular disease in people with type 2 diabetes: the Edinburgh Type 2 Diabetes Study. Diabetologia 201053 467-471. (doi:10.1007/ s00125-009-1628-9)

11 Reynolds RM, Strachan MW, Labad J, Lee AJ, Frier BM, Fowkes FG, Mitchell R, Seckl JR, Deary IJ, Walker BR \& Price JF \& Edinburgh Type 2 Diabetes study investigators. Morning cortisol levels and cognitive abilities in people with type 2 diabetes: the Edinburgh Type 2 Diabetes Study. Diabetes Care 201033 714-720. (doi:10.2337/dc09-1796)

12 Blackburn D, Hux J \& Mamdani M. Quantification of the risk of corticosteroid-induced diabetes mellitus among the elderly. Journal of General Internal Medicine $2002 \quad \mathbf{1 7} \quad 717-720$. (doi:10.1046/j.1525-1497.2002.10649.x)

13 Gulliford MC, Charlton J \& Latinovic R. Risk of diabetes associated with prescribed glucocorticoids in a large population. Diabetes Care 200629 2728-2729. (doi:10.2337/dc06-1499)

14 Gurwitz JH, Bohn RL, Glynn RJ, Monane M, Mogun H \& Avorn J. Glucocorticoids and the risk for initiation of hypoglycemic therapy. Archives of Internal Medicine $1994 \mathbf{1 5 4}$ 97-101. (doi:10.1001/ archinte.1994.00420010131015)
15 Price JF, Reynolds RM, Mitchell RJ, Williamson RM, Fowkes FG, Deary IJ, Lee AJ, Frier BM, Hayes PC \& Strachan MW. The Edinburgh Type 2 Diabetes Study: study protocol. BMC Endocrine Disorders 20088 18. (doi:10.1186/1472-6823-8-18)

16 Rose GA. The diagnosis of ischaemic heart pain and intermittent claudication in field surveys. Bulletin of the World Health Organisation 196227 645-658.

17 Gale CR, Allerhand M, Sayer AA, Cooper C, Dennison EM, Starr JM, Ben-Shlomo Y, Gallacher JE, Kuh D \& Deary IJ \& HALCyon Study Team. The structure of the Hospital Anxiety and Depression Scale in four cohorts of community-based, healthy older people: the HALCyon program. International Psychogeriatrics 201022 559-571. (doi:10.1017/S1041610210000256)

18 Bjelland I, Dahl AA, Haug TT \& Neckelmann D. The validity of the Hospital Anxiety and Depression Scale. An updated literature review. Journal of Psychosomatic Research 200252 69-77. (doi:10.1016/S0022-3999(01)00296-3)

19 Reynolds RM, Labad J, Strachan MW, Braun A, Fowkes FG, Lee AJ, Frier BM, Seckl JR, Walker BR \& Price JF \& Edinburgh Type 2 Diabetes Study (ET2DS) Investigators. Elevated fasting plasma cortisol is associated with ischemic heart disease and its risk factors in people with type 2 diabetes: the Edinburgh Type 2 Diabetes Study. Journal of Clinical Endocrinology and Metabolism 201095 1602-1608. (doi:10.1210/jc.2009-2112)

20 Williamson RM, Price JF, Glancy S, Perry E, Nee LD, Hayes PC, Frier BM, Van Look LA, Johnston GI, Reynolds RM \& Strachan MW $\&$ Edinburgh Type 2 Diabetes study investigators. Prevalence of and risk factors for hepatic steatosis and nonalcoholic fatty liver disease in people with type 2 diabetes: the Edinburgh Type 2 Diabetes Study. Diabetes Care 201134 1139-1144. (doi:10.2337/ dc10-2229)

21 Williamson RM, Perry E, Glancy S, Marshall I, Gray C, Nee LD, Hayes PC, Forbes S, Frier BM, Johnston GI, Lee AJ, Reynolds RM, Price JF, MW Strachan \& Edinburgh Type 2 Diabetes study investigators. The use of ultrasound to diagnose hepatic steatosis in type 2 diabetes: intra- and interobserver variability and comparison with magnetic resonance spectroscopy. Clinical Radiology 201166 434-439. (doi:10.1016/j.crad.2010.09.021)

22 Walker BR. Glucocorticoids and cardiovascular disease. European Journal of Endocrinology 2007157 545-559. (doi:10.1530/EJE07-0455)

23 Rockall AG, Sohaib SA, Evans D, Kaltsas G, Isidori AM, Monson JP, Besser GM, Grossman AB \& Reznek RH. Hepatic steatosis in Cushing's syndrome: a radiological assessment using computed tomography. European Journal of Endocrinology 2003 149 543-548. (doi:10.1530/eje.0.1490543)

24 Katon W, Von Korff M, Ciechanowski P, Russo J, Lin E, Simon G, Ludman E, Walker E, Bush T \& Young B. Behavioral and clinical factors associated with depression among individuals with diabetes. Diabetes Care 200427 914-920. (doi:10.2337/diacare.27. 4.914)

25 Taylor VA, Ellenbogen MA, Washburn D \& Joober R. The effects of glucocorticoids on the inhibition of emotional information: a dose-response study. Biological Psychology 201186 17-25. (doi:10.1016/j.biopsycho.2010.10.001)

26 Finkel D, Reynolds CA, McArdle JJ \& Pedersen NL. Age changes in processing speed as a leading indicator of cognitive aging. Psychology and Aging 200722 558-568. (doi:10.1037/08827974.22.3.558)

27 Stranahan AM, Arumugam TV, Cutler RG, Lee K, Egan JM \& Mattson MP. Diabetes impairs hippocampal function through glucocorticoid-mediated effects on new and mature neurons. Nature Neuroscience 200811 309-317. (doi:10.1038/nn2055)

Received 16 January 2012

Revised version received 25 February 2012

Accepted 9 March 2012 